\title{
A Nuclear Magnetic Resonance and Fourier-Transform Infrared Spectroscopic Study of Lyophilized Umbilical Cord Blood Platelet Rich Plasma
}

\author{
Serene Joy ${ }^{1 \mathbb{D}}$, Ashmitha Kishan Shetty ${ }^{1, * \mathbb{D}}$, Rajamanickam Deveswaran ${ }^{2} \mathbb{( D )}$, Jayaraman Anbu ${ }^{3(\mathbb{D})}$, \\ Manasa Biligowda Latha ${ }^{1}$ (D), Shivani Selvappa ${ }^{1}$ (D), Siddique Sha Muhammed Hussain ${ }^{1(D)}$ \\ 1 Department of Pedodontics and Preventive Dentistry, Faculty of Dental Sciences, M.S. Ramaiah University of Applied \\ Sciences, India; dr.serenerosa@gmail.com $\quad$ (S.J.); $\quad$ ashmitha.pe.ds@msruas.ac.in $\quad$ (A.K.S.); \\ manasabgowda1208@gmail.com (M.B.L.); shivanicheluvaraj13@gmail.com (S.S.); siddiquesha17@gmail.com \\ (S.S.M.H); \\ 2 Department of Pharmaceutics, Faculty of Pharmacy, M.S. Ramaiah University of Applied Sciences, India; \\ deveswaran.ps.ph@msruas.ac.in (R.D.); \\ 3 Department of Pharmacology, Faculty of Pharmacy, M.S. Ramaiah University of Applied Sciences, India; \\ anbu.pg.ph@msruas.ac.in (J.A.); \\ * Correspondence: ashmitha.pe.ds@msruas.ac.in (A.K.S.);
}

Scopus Author ID 56465464300

Received: 7.04.2021; Revised: 15.08.2021; Accepted: 18.08.2021; Published: 12.09.2021

Abstract: Cord blood platelet-rich plasma is regarded as a potential therapeutic agent with wound healing and regenerative purposes. It has many advantages, such as availability, universal use, and being rich in regenerative tissue factors. To characterize the Umbilical Cord Blood Platelet Rich Plasma (UCB-PRP), umbilical cord blood is collected from healthy mothers in sterile vacutainers. Platelet-rich plasma was extracted after two sets of centrifugations and lyophilized using the freeze-drying method. The samples are subjected to physicochemical characterization using Nuclear Magnetic Resonance (NMR) and Fourier-Transform Infrared (FTIR) Spectroscopy. This translational approach provided evidence of clinical effectiveness for understanding physiology and pathophysiology at the molecular level. Accordingly, FTIR and NMR studies provided information on the structural stability of protein molecules present in UCB-PRP.

Keywords: UCB; PRP; FT-IR; NMR.

(C) 2021 by the authors. This article is an open-access article distributed under the terms and conditions of the Creative Commons Attribution (CC BY) license (https://creativecommons.org/licenses/by/4.0/).

\section{Introduction}

The whole blood is a complex fluid and a combination of cells, colloids, and crystalloids. Different blood products, such as Packed Red Blood Cell (PRBC) concentrate, platelet concentrate, fresh frozen plasma, and cryoprecipitate, can be isolated from the formed elements of blood. PRBCs have the highest specific gravity, followed by leucocytes, platelets, and plasma. The isolation of the components is achieved by centrifuging one unit of entire blood [1]. For therapeutic use, blood is processed into two sets: (i) Labile Blood Components (LBCs) are made up mostly of cellular components (Red Blood Cell Concentrates (RBCCs) and Platelet Concentrates (PCs)), as well as a portion of therapeutic plasma, primarily Fresh Frozen Plasma (FFP). The other category includes (ii)substances extracted from plasma or fractionated drugs, as well as FFP, acquired from vast reservoirs and subjected to extensive pathogen reduction [2]. 
Platelet-Rich Plasma (PRP) is a small portion of autologous blood plasma that has been saturated with platelets from the blood. PRP is considered to assist in various healing mechanisms due to the growth factors present in platelet alpha-granules. The growth factors act as signaling molecules for the cells. Because of the main beneficial actions that PRP possess, its use is being investigated extensively for clinical applications in various fields in dentistry and medicine such as periodontology and oral and maxillofacial surgery, cosmetic surgery, and in treatment of wound healing of chronic ulcers of the skin and soft tissue [3].

Although both adult Peripheral Blood (PB) and Umbilical Cord Blood (UCB) can be used to make PRP, research indicates that the form and volume of factors in each vary considerably. In comparison to PB PRP, UCB-PRP has been shown to have better therapeutic qualities as the former has high levels of pro-inflammatory molecules that cause aggravation of inflammatory responses, while UCB-PRP contains high levels of anti-inflammatory factors. Furthermore, UCB-PRP comprises a number of Growth Factors (GF), cytokines, and other immunomodulatory factors that induce cell proliferation and differentiation. Centrifugation of PRP helps to enhance the accumulation and lysis of platelets, resulting in the release of growth factors such as Platelet-Derived Growth Factor (PDGF), basic Fibroblast Growth Factor (b-FGF), Epithelial Growth Factor (EGF), Vascular Endothelial Growth Factor (VEGF), and Transforming Growth Factor-beta (TGF- $\beta$ ), among others, all of which have been shown to improve tissue repair and regeneration [4]. Due to these added advantages, UCB has extensive use in the field of medicine. Umbilical cord blood serum is recently marketed as eyedrops for the treatment of keratinized cornea [5]. Certain growth factors are present in the cord blood serum, which exhibit unique differentiation potential and have pharmacological influence to boost anti-inflammatory, analgesic, and wound healing properties[6].

Teeth are complex mineralized organs consisting of distinctive tissues, enamel, dentin, cementum, and pulp, supported by the surrounding alveolar bone. Pulp exposure from caries, trauma, or tooth preparation mishaps can lead to severe pain with resultant infection. The consequences of treating pulp exposures are often extraction or root canal therapy. Multiple appointments are invested in both loss of teeth followed by providing prosthesis as well as endodontic treatment. Tooth regeneration epitomizes a revolution in stomatology as a paradigm shift from conventional repair to regeneration: regeneration provides means for biological restoration [7].

Drug Delivery Systems (DDS) are pharmaceutical formulations that safely and effectively deliver prescription medications and bioactive compounds throughout the body as needed to achieve the desired therapeutic effect [8]. A variety of targeted drug delivery modalities have recently been demonstrated, and drug modulation by chemical means, drug release using scaffolds, and other techniques are among them; to use a protein as a drug, care needs to be taken about the long-term stability of the protein. The long-lasting sustainability of protein therapeutics in the solid state is contingent on preserving the original composition during lyophilization and in the lyophilized compound. Characterizing proteins in their dried state is thus necessary for the development of secure as well as efficient formulations[9].

Fourier Transform Infrared (FTIR) spectroscopy can characterize and quantity the echelons of complex biomolecules present in biological samples, resulting in a biochemical fingerprint due to the consequent spectrum. When combined with statistical processing and machine learning algorithms, the technique has shown high precision in identifying subtle differences in blood composition [10]. 
Nuclear Magnetic Resonance (NMR) provides a quicker and more comprehensive evaluation of biological samples in human models. NMR-based untargeted metabolomics has been an excellent replacement in disease research to characterize biomarkers for early intervention and treatment detection, accurate prognosis, and disease progression monitoring [11].

NMR and FTIR spectrometry are two major physicochemical techniques used to analyze important insights into the molecular data in the biological sample contents. Thus, the study aims to characterize the UCB-PRP using NMR and FTIR.

\section{Materials and Methods}

\subsection{Preparation of lyophilized UCB-PRP.}

Fresh full-term human umbilical cord (40 weeks gestation period) within 30 minutes of delivery was obtained from 30 healthy mothers. The informed consent was taken from University Ethics Committee for Human Trials. The inclusion criteria were that the umbilical cord blood would be obtained from a C-section from healthy mothers. The exclusion criteria were Intrauterine growth impairment, fetal defects, hypertension, anemia, diabetes, tobacco or drug abuse, induced or early labor, pregnancy complications, and other medical or obstetric risks. The blood was collected in a $50 \mathrm{ml}$ sterile vacutainer coated with anticoagulants. The obtained blood was immediately subjected to two sets of centrifugations. The first centrifugation was for $1400 \mathrm{rpm}$ for 10 minutes at $37^{\circ} \mathrm{C}$. The serum separated from the blood was subjected to the second centrifugation for $2400 \mathrm{rpm}$ for 15 minutes at $37^{\circ} \mathrm{C}$. The platelets segregated at the bottom of the tube were homogenized, with one-third of the platelet-poor plasma formed on top [12]. The obtained sample was pre-frozen at $-80^{\circ} \mathrm{C}$ for 24 hours before subjecting it to the freeze-drying process and lyophilized. PRP powder was stored at $-20^{\circ} \mathrm{C}[6]$.

\subsection{FTIR spectroscopy.}

The lyophilized biomaterial was analyzed by FTIR spectrometer (FTIR - 8400S instrument). The spectrum was assessed for 10 scans from $400-4000 \mathrm{~cm}^{-1}$ at a resolution of 4 $\mathrm{cm}^{-1}$ in transmittance mode [13]. HyperIR Software was used for determining the infrared spectroscopy of the sample. The obtained peak was smoothened as per the requirement.

\subsection{NMR analysis.}

1D ${ }^{1} \mathrm{H}$ NMR spectra of lyophilized UCB-PRP were analyzed at $298 \mathrm{~K}$ on a Bruker AV $800 \mathrm{MHz}$ spectrometer operating at $800.103 \mathrm{MHz}$. The NMR spectrum was recorded using the $\mathrm{D}_{2} \mathrm{O}$-presaturated standard one-dimensional zgesgp pulse sequence. The ${ }^{1} \mathrm{H}$ NMR standard 1$\mathrm{D}$ analysis was attained with a sweep width of $20 \mathrm{ppm}$, ensuing in an acquisition time of 1.49 s. The experiments were achieved with 128 scans, 2 dummy scans at a temperature of $298 \mathrm{~K}$ operating in a spectral width of $16 \mathrm{kHz}$ with a relaxation delay of $2 \mathrm{~s}$ [14].

\section{Results and Discussion}

The infrared spectra showed ranging from $3456-437 \mathrm{~cm}^{-1}$ for UCB-PRP. The amide I band $\left(1620 \mathrm{~cm}^{-1}\right.$ and $\left.1652 \mathrm{~cm}^{-1}\right)$ indicating the $\mathrm{C}=\mathrm{O}$ group and amide II band $\left(1514 \mathrm{~cm}^{-1}\right.$ and $1544 \mathrm{~cm}^{-1}$ ) indicative of $\mathrm{N}-\mathrm{H}$ group and a wideband between $3276 \mathrm{~cm}^{-1}$ and $3456 \mathrm{~cm}^{-1}$ 
indicative of - $\mathrm{OH}$ group.2921-2956 $\mathrm{cm}^{-1}$ indicates the presence of long-chain fatty acids and phospholipids (Figure 1).

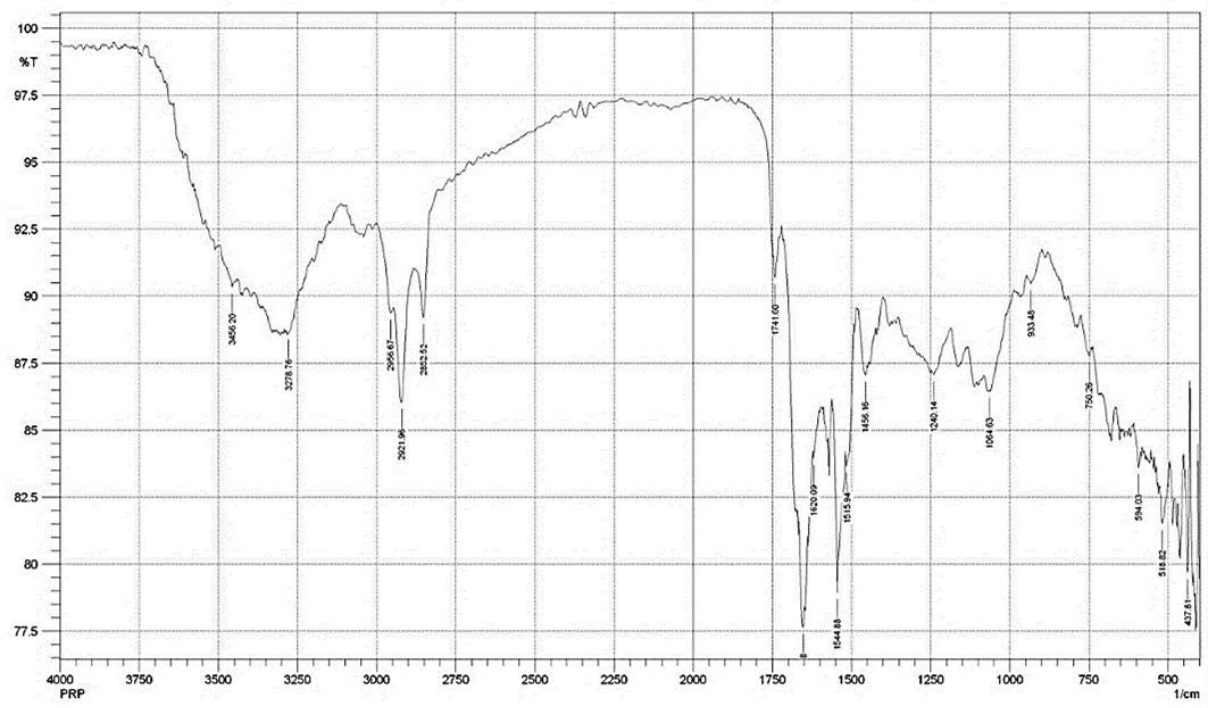

Figure 1. FT-IR spectra of freeze-dried umbilical cord blood platelet-rich plasma show prominent peaks at $1515,1544,1620,2852,2921,2956$ and a broad area from 3278-3456.

Table 1. Peak values obtained after the freeze-dried UCB-PRP are subjected to FTIR.

\begin{tabular}{c|c|c|c|c|c|c|c} 
& Peak & Height & Corr.Height & Base $(\mathbf{H})$ & Base $(\mathbf{L})$ & Area & Corr.Area \\
\hline $\mathbf{1}$ & 437.81 & 20.321 & 6.333 & 451.31 & 432.03 & 1.628 & 0.325 \\
\hline $\mathbf{2}$ & 518.82 & 18.49 & 1.551 & 526.53 & 495.67 & 2.591 & 0.151 \\
\hline $\mathbf{3}$ & 594.03 & 16.398 & 0.896 & 609.46 & 588.25 & 1.571 & 0.04 \\
\hline $\mathbf{4}$ & 750.26 & 12.232 & 0.723 & 775.33 & 738.69 & 1.99 & 0.077 \\
\hline $\mathbf{5}$ & 933.48 & 9.539 & 0.59 & 946.98 & 898.77 & 1.985 & 0.075 \\
\hline $\mathbf{6}$ & 1064.63 & 13.549 & 0.213 & 1068.49 & 987.49 & 4.378 & 0.049 \\
\hline $\mathbf{7}$ & 1240.14 & 12.937 & 0.376 & 1247.86 & 1186.14 & 3.461 & 0.049 \\
\hline $\mathbf{8}$ & 1456.16 & 12.948 & 1.037 & 1479.3 & 1446.51 & 1.853 & 0.105 \\
\hline $\mathbf{9}$ & 1515.94 & 16.333 & 1.222 & 1519.8 & 1492.8 & 1.86 & 0.19 \\
\hline $\mathbf{1 0}$ & 1544.88 & 21.046 & 6.41 & 1562.23 & 1519.8 & 3.559 & 0.593 \\
\hline $\mathbf{1 1}$ & 1620.09 & 16.062 & 0.429 & 1622.02 & 1598.88 & 1.63 & 0.012 \\
\hline $\mathbf{1 2}$ & 1652.88 & 22.349 & 3.818 & 1670.24 & 1639.38 & 3.118 & 0.375 \\
\hline $\mathbf{1 3}$ & 1741.6 & 9.316 & 2.826 & 1805.25 & 1722.31 & 2.163 & 0.273 \\
\hline $\mathbf{1 4}$ & 2852.52 & 10.799 & 2.962 & 2881.45 & 2806.23 & 2.815 & 0.294 \\
\hline $\mathbf{1 5}$ & 2921.96 & 13.967 & 4.126 & 2947.03 & 2883.38 & 3.325 & 0.515 \\
\hline $\mathbf{1 6}$ & 2956.67 & 10.629 & 0.673 & 2999.1 & 2948.96 & 2.05 & 0.041 \\
\hline $\mathbf{1 7}$ & 3278.76 & 11.363 & 0.257 & 3284.55 & 3244.05 & 1.995 & 0.04 \\
\hline $\mathbf{1 8}$ & 3456.2 & 9.66 & 0.36 & 3494.77 & 3450.41 & 1.824 & 0.044
\end{tabular}

The standard 1D ${ }^{1} \mathrm{H}$ NMR analysis data of the lyophilized UCB-PRP showed values ranging between $\sim 1-4$, signifying the aggregation of macromolecules and metabolites present in the biomaterial, indicating the presence of aliphatic group ( 1-2) and hydroxyl group ( 34).

Platelets aid with hemostasis, tissue regeneration, and host protection.PRP and its derivatives are used medically to facilitate wound healing in many circumstances dependent on these conditions [15]. Both dental and medical fields have also seen progress with autologous peripheral blood platelet derivatives. UCB-platelet derivatives are a significant substitute to autologous equivalents, such as in the case of an autologous contraindication for the development of platelet derivatives due to patient rejection or pain, inadequacy of venous access, or in conjunction with inflammatory, autoimmune or hematological diseases [16]. The proteomic profile of Platelet gel released and Platelet gel collected from either PB or UCB has been extensively compared in a recent study. Large variations in two bio factor clusters were 
detected after multi-analyte characterization (188 biomarkers) was performed. It was noticed that UCB possesses higher angiogenic factors such as resistin, prolactin, VEGF, growth hormone, trefoil factor 3, and erythropoietin concentrations compared to PB. At the same time, PB was abundant in inflammatory as well as immunomodulatory factors such as CD5 antigenlike, chemokine HCC-4, MMP-3, IgA, IgM, and RANTES [17].

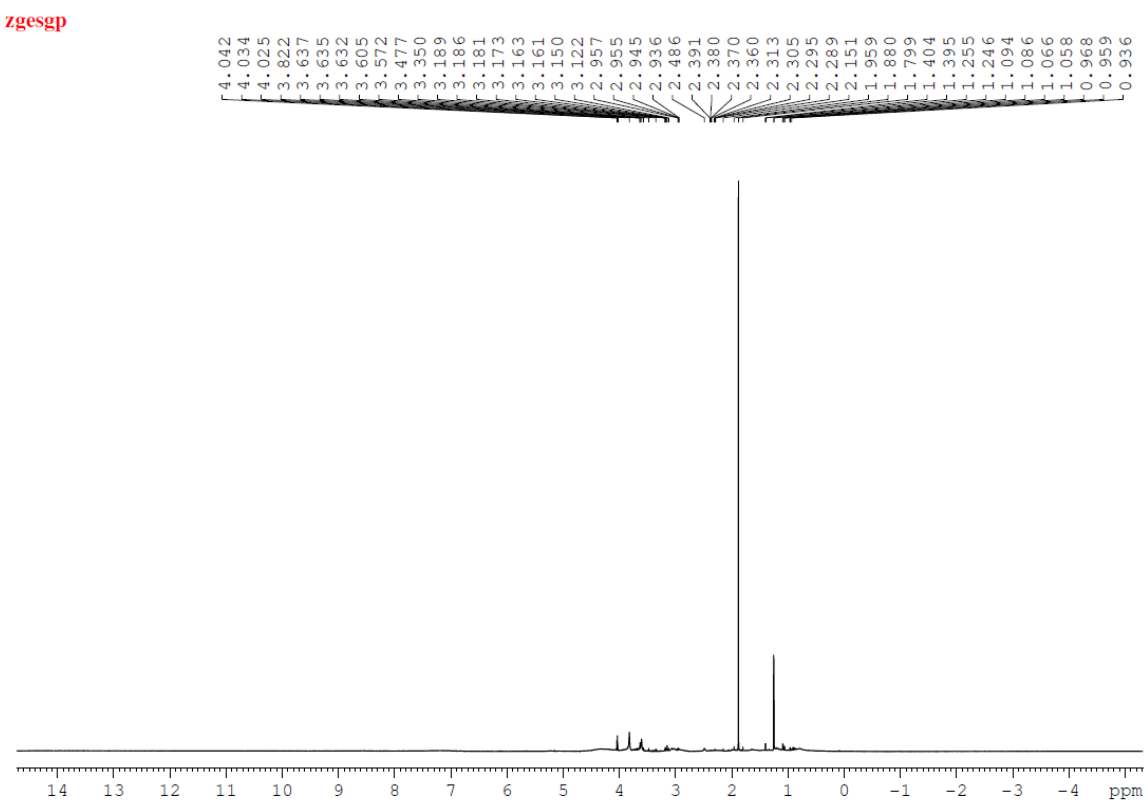

Figure 2. 1D ${ }^{1} \mathrm{H}-\mathrm{NMR}$ spectra of lyophilized umbilical cord platelet-rich plasma, the spectra ranges from $\sim 1-4$.

Fourier Transform Infrared Spectroscopy through Attenuated Total Reflectance (FTIRATR) has been used for many biological specimen studies. The latest applications include a study of the whole blood and analyses of its fractions, protein, or lipid components. The amide I band is instigated by the peptide bond's carbonyl $(\mathrm{C}=\mathrm{O})$ stretching vibrations, while the amide II band is produced by the $\mathrm{C}-\mathrm{N}$ stretching vibrations and $\mathrm{N}-\mathrm{H}$ bending modes. Protein secondary structure is observed by both bands [18]. The classic characterization approach to determine the chemical composition of the secondary structure and the functional groups is infrared spectroscopic analysis. The peak ranges from $3002 \mathrm{~cm}-1$ to $3400 \mathrm{~cm}-1$ reflect the stretching vibration of hydroxyl(-OH) bonds, shown in a similar study conducted in PRP. The characteristic PRP peaks of amide III and amide A were at $1400 \mathrm{~cm}^{-1}$ and $3270 \mathrm{~cm}^{-1}$, respectively. The amide A $\left(3270 \mathrm{~cm}^{-1}\right)$ band is related to the stretching vibrations of the N-H and O-H pairs [13]. Another research found identical FTIR spectra of PRP, suggesting that the biomaterial's functional group was maintained after lyophilization [19]. This indicates that factors apart from the global secondary structure retentivity as assessed by FTIR affect solidstate protein stability [9].

The chemical structure of the biocompatible chitosan-collagen-hydroxyapatite nanofibers encrusted with platelet-rich plasma when investigated by FTIR spectroscopy have exhibited amide I and II bands at similar peaks from the present study [19]. On the quantitative lipoprotein and metabolite forms, results with varying sizes of blood storage tubes and consecutive freeze-thaw cycles, preservatives, sample storage at $-80{ }^{\circ} \mathrm{C}$, short-term storing at $4^{\circ} \mathrm{C}$, and also at $20{ }^{\circ} \mathrm{C}$ were examined [20].

As per our knowledge, this is the first research to use $1 \mathrm{D}{ }^{1} \mathrm{H}-\mathrm{NMR}$ to analyze the UCBPRP. In plasma, almost all molecules contain hydrogen. In theory, this may mean that the 1D ${ }^{1} \mathrm{H}$ NMR spectrum would display signals from all plasma metabolites[21]. NMR can be used to evaluate any form of a biological matrix with minimal sample manipulation in terms of 
samples [22]. The recognition of an amassed number of metabolites has ignited interest in combining the strengths of NMR and Mass Spectrometry methods for unidentified metabolite detection, biomarker discovery, and direct assessment of enormous amounts of metabolomics literature produced using the two prevailing methods [23].

The limitation of FTIR is the fact that FTIR only detects global protein conformation and not subpopulations of local conformational changes that can impact stability is a drawback of the technique. FTIR is often incapable of distinguishing aggregation produced by variations in tertiary structure, emphasizing the need for improved solid-state resolution. The sensitivity of NMR is inadequate when compared to Mass Spectrometry, another drawback of NMR analysis. 1D ${ }^{1} \mathrm{H}$ NMR spectra are seen to be crowded and full of chemical details as the hydrogen atom is the most sensitive nuclei, which is present abundantly in the bio-sample.

\section{Conclusions}

Correspondence between the findings attained using FTIR spectroscopy and $1 \mathrm{D}{ }^{1} \mathrm{H}$ NMR system of lyophilized UCB-PRP gives the information that the alkyl group and the carbonyl group indicate many viable proteins readily available for the interaction with tissue. Future work will include characterizing the sample biomaterial to identify these active proteins and assessing the potential of this UCB-PRP in the field of regenerative medicine.

\section{Funding}

This article received no external funding.

\section{Acknowledgments}

We acknowledge the support from Lakshmi Maternity Home, Bangalore, for providing us with cord blood samples and NMR Research Centre, Indian Institute of Science, Bangalore, for their valuable help in analyzing the biomaterial.

\section{Conflicts of Interest}

The authors declare no conflict of interest.

\section{References}

1. Basu, D.; Kulkarni, R. Overview of Blood Components and Their Preparation. Indian J Anaesth 2014, 58, 529-537, https://doi.org/10.4103/0019-5049.144647.

2. Garraud, O.; Tissot, J.-D. Blood and Blood Components: From Similarities to Differences. Front. Med. 2018, 5, https://doi.org/10.3389/fmed.2018.00084.

3. Oudelaar, B.W.; Peerbooms, J.C.; Huis In't Veld, R.; Vochteloo, A.J.H. Concentrations of Blood Components in Commercial Platelet-Rich Plasma Separation Systems: A Review of the Literature. Am J Sports Med 2019, 47, 479-487, https://doi.org/10.1177/0363546517746112.

4. Samarkanova, D.; Cox, S.; Hernandez, D.; Rodriguez, L.; Casaroli-Marano, R.P.; Madrigal, A.; Querol, S. Cord Blood Platelet Rich Plasma Derivatives for Clinical Applications in Non-Transfusion Medicine. Front Immunol 2020, 11, https://doi.org/10.3389/fimmu.2020.00942.

5. Giannaccare, G.; Carnevali, A.; Senni, C.; Logozzo, L.; Scorcia, V. Umbilical Cord Blood and Serum for TheTreatment of Ocular Diseases: A Comprehensive Review. Ophthalmol Ther 2020, 9, 235-248, https://doi.org/10.1007/s40123-020-00239-9.

6. Yassin, G.E.; Dawoud, M.H.S.; Wasfi, R.; Maher, A.; Fayez, A.M. Comparative Lyophilized Platelet-Rich Plasma Wafer and Powder for Wound-Healing Enhancement: Formulation, in Vitro and in Vivo Studies. 
Drug Development and Industrial Pharmacy 2019, 45, 1379-1387, https://doi.org/10.1080/03639045.2019.1620269.

7. Yildirim, S.; Fu, S.Y.; Kim, K.; Zhou, H.; Lee, C.H.; Li, A.; Kim, S.G.; Wang, S.; Mao, J.J. Tooth Regeneration: A Revolution in Stomatology and Evolution in Regenerative Medicine. International Journal of Oral Science 2011, 3, 107-116, https://doi.org/10.4248/IJOS11042.

8. Damiri, F.; Bachra, Y.; Bounacir, C.; Laaraibi, A.; Berrada, M. Synthesis and Characterization of Lyophilized Chitosan-Based Hydrogels Cross-Linked with Benzaldehyde for Controlled Drug Release. Journal of Chemistry 2020, 2020, https://doi.org/10.1155/2020/8747639.

9. Moorthy, B.S.; Iyer, L.K.; Topp, E.M. Characterizing Protein Structure, Dynamics and Conformation in Lyophilized Solids. Curr Pharm Des 2015, 21, 5845-5853, https://doi.org/10.2174/1381612821666151008150735.

10. Cameron, J.M.; Butler, H.J.; Anderson, D.J.; Christie, L.; Confield, L.; Spalding, K.E.; Finlayson, D.; Murray, S.; Panni, Z.; Rinaldi, C.; Sala, A.; Theakstone, A.G.; Baker, M.J. Exploring Pre-Analytical Factors for the Optimisation of Serum Diagnostics: Progressing the Clinical Utility of ATR-FTIR Spectroscopy. Vibrational Spectroscopy 2020, 109, 103092, https://doi.org/10.1016/j.vibspec.2020.103092.

11. Silva, R.A.; Pereira, T.C.S.; Souza, A.R.; Ribeiro, P.R. 1H NMR-Based Metabolite Profiling for Biomarker Identification. Clinica Chimica Acta 2020, 502, 269-279, https://doi.org/10.1016/j.cca.2019.11.015.

12. da Silva, L.Q.; Montalvão, S.A. de L.; Justo-Junior, A. da S.; Cunha Júnior, J.L.R.; Huber, S.C.; Oliveira, C.C.; Annichino-Bizzacchi, J.M. Platelet-Rich Plasma Lyophilization Enables Growth Factor Preservation and Functionality When Compared with Fresh Platelet-Rich Plasma. Regenerative Medicine 2018, 13, 775784, https://doi.org/10.2217/rme-2018-0035.

13. Lei, X.; Yang, Y.; Shan, G.; Pan, Y.; Cheng, B. Preparation of ADM/PRP Freeze-Dried Dressing and Effect of Mice Full-Thickness Skin Defect Model. Biomed Mater 2019, 14, 035004, https://doi.org/10.1088/1748$605 X / a b 0060$.

14. Cheng, P.; Xue, X.; Su, J.; Lu, M.; Wang, S.; Meng, J. 1H NMR-Based Metabonomic Revealed Protective Effect of Moutan Cortex Charcoal on Blood-Heat and Hemorrhage Rats. Journal of Pharmaceutical and Biomedical Analysis 2019, 169, 151-158, https://doi.org/10.1016/j.jpba.2019.02.044.

15. Oneto, P.; Etulain, J. PRP in Wound Healing Applications. Platelets 2020, 32, 189-199, https://doi.org/10.1080/09537104.2020.1849605.

16. Orlando, N.; Pellegrino, C.; Valentini, C.G.; Bianchi, M.; Barbagallo, O.; Sparnacci, S.; Forni, F.; Fontana, T.M.; Teofili, L. Umbilical Cord Blood: Current Uses for Transfusion and Regenerative Medicine. Transfusion and Apheresis Science 2020, 59, 102952, https://doi.org/10.1016/j.transci.2020.102952.

17. Parazzi, V.; Lavazza, C.; Boldrin, V.; Montelatici, E.; Pallotti, F.; Marconi, M.; Lazzari, L. Extensive Characterization of Platelet Gel Releasate From Cord Blood in Regenerative Medicine. 2015, 24, 2573-2584, https://doi.org/10.3727/096368915X687471.

18. Bujok, J.; Gąsior-Głogowska, M.; Marszałek, M.; Trochanowska-Pauk, N.; Zigo, F.; Pavl’ak, A.; Komorowska, M.; Walski, T. Applicability of FTIR-ATR Method to Measure Carbonyls in Blood Plasma after Physical and Mental Stress. BioMed Research International 2019, 2019, https://doi.org/10.1155/2019/2181370.

19. Tang, Y.; Zhang, H.; Wei, Q.; Tang, X.; Zhuang, W. Biocompatible Chitosan-Collagen-Hydroxyapatite Nanofibers Coated with Platelet-Rich Plasma for Regenerative Engineering of the Rotator Cuff of the Shoulder. RSC Advances 2019, 9, 27013-27020, https://doi.org/10.1039/C9RA03972D.

20. Loo, R.L.; Lodge, S.; Kimhofer, T.; Bong, S.-H.; Begum, S.; Whiley, L.; Gray, N.; Lindon, J.C.; Nitschke, P.; Lawler, N.G.; Schafer, H.; Spraul, M.; Richards, T.; Nicholson, J.K.; Holmes, E. Quantitative In-Vitro Diagnostic NMR Spectroscopy for Lipoprotein and Metabolite Measurements in Plasma and Serum: Recommendations for Analytical Artifact Minimization with Special Reference to COVID-19/SARS-CoV-2 Samples. J. Proteome Res. 2020, 19, 4428-4441, https://doi.org/10.1021/acs.jproteome.0c00537.

21. Ala-Korpela, M. 1H NMR Spectroscopy of Human Blood Plasma. Progress in Nuclear Magnetic Resonance Spectroscopy 1995, 27, 475-554, https://doi.org/10.1016/0079-6565(95)01013-0.

22. Takis, P.G.; Ghini, V.; Tenori, L.; Turano, P.; Luchinat, C. Uniqueness of the NMR Approach to Metabolomics. TrAC Trends in Analytical Chemistry 2019, 120, 115300, https://doi.org/10.1016/j.trac.2018.10.036.

23. Gowda, G.A.N.; Raftery, D. Quantitating Metabolites in Protein Precipitated Serum Using NMR Spectroscopy. Anal. Chem. 2014, 86, 5433-5440, https://doi.org/10.1021/ac5005103. 